\title{
Phenotyping chronic obstructive pulmonary disease (COPD) exacerbations: realising personalised medicine
}

\author{
Chris E Brightling and Bethan L Barker
}

\section{Introduction}

Chronic obstructive pulmonary disease (COPD) is characterised by airflow obstruction (post-bronchodilator forced expiratory volume in one second $\left(\mathrm{FEV}_{1}\right)$ /forced vital capacity (FVC) ratio $<0.7)$ that is not fully reversible, does not change markedly over several months and is usually progressive. The main causative agent is cigarette smoke, although other exposures (for example, occupational and biomass fuel) are increasingly recognised to be important. Stable disease is punctuated by exacerbations, which are an acute onset of sustained worsening of the patient's symptoms from their usual stable state beyond normal day-to-day variations. Commonly reported symptoms are worsening breathlessness, cough, increased sputum production and change in sputum colour. The change in these symptoms often necessitates a change in medication. ${ }^{1}$

Chronic obstructive pulmonary disease is an important cause of morbidity and mortality and in the UK is responsible for about 30,000 deaths per year, $10 \%$ of all hospital bed-days and $>£ 800$ million per year in direct healthcare costs. Exacerbations are particularly important, as they are associated with high economic costs and accelerated decline in lung function and have a negative impact on quality of life and mortality. By 2030, COPD is predicted to become the third leading cause of death worldwide. ${ }^{1}$

Guidelines for the treatment of stable COPD recommend a stratified approach to treatment determined by disease severity (defined by spirometry), with stepwise increases in treatment as the disease progresses. This treatment approach includes smoking cessation, bronchodilators, inhaled corticosteroids, pneumococcal and influenza immunisations, and exercise in the form of pulmonary rehabilitation. ${ }^{1}$ Current guidelines recommend treatment of exacerbations with corticosteroids, together with antibiotics in the presence of increased sputum production and purulence.

Recognition that COPD is a heterogeneous condition with variable response to current therapies is increasing. Indeed, although therapy improves symptoms and exercise capacity and reduces exacerbation frequency, the magnitude of benefit is small and restricted to subgroups of patients, and none of the current therapies affect disease progression. We therefore need to improve our understanding of the gene-environment

Chris E Brightling, professor of respiratory medicine and Wellcome Trust senior clinical fellow; Bethan L Barker, clinical research fellow

Institute for Lung Health, Department of Infection, Immunity and Inflammation, University of Leicester, UK interactions that underlie the interplay between pollutants, host susceptibility to damage, remodelling and persistent infection, and the development of exacerbations in order to better stratify and personalise current and future therapies.

\section{Phenotyping stable COPD}

A phenotype is any observable characteristic that results from gene-environment interactions. Phenotyping needs to consider information derived at different scales of disease - ie gene-cell, cell-tissue, tissue-organ and organ-whole person interactions (Fig 1). For example, patients can be phenotyped at the wholeperson scale on the basis of their symptoms, such as chronic bronchitis versus breathlessness, and at the organ scale on the basis of lung function, or radiologically by low attenuation areas on computed tomography (CT) images consistent with emphysema versus bronchial wall thickening suggestive of airways disease. At the cell-tissue scale, non-invasive measures of airway inflammation such as sputum analysis can determine inflammatory phenotypes defined by cellular composition or inflammatory mediators. This approach has been very informative and has revealed that most airway inflammation in COPD is neutrophilic, but eosinophil levels in sputum are persistently increased in the stable state and during exacerbations in a subgroup of patients. ${ }^{2}$ At the gene-cell scale, the best example of genetic variation in COPD is alpha 1-antitrypsin deficiency, with the $\mathrm{ZZ}$ genotype predisposing to early-onset emphysema in Caucasian people. Intriguingly, only a proportion (about 30\%) of smokers develop COPD, which supports the concept that it is an environmental exposure combined with host/genetic factors that lead to disease development. Recent genomewide association studies have identified important single nucleotide polymorphisms associated with airflow obstruction, including glutathione S-transferase D and receptor for advanced glycation end-products (RAGE), which implicates oxidative stress and damage-recognition receptors.

Most approaches to phenotyping consider disease at a single scale and do not integrate information to provide multidimensional phenotypes. An approach that integrates the underlying biology of the disease with clinical expression is critical to inform understanding of the disease and to help define subgroups to target therapies.

\section{Phenotyping COPD exacerbations}

The most likely cause of COPD exacerbations is infection of the airway with viruses and bacteria, although the association 


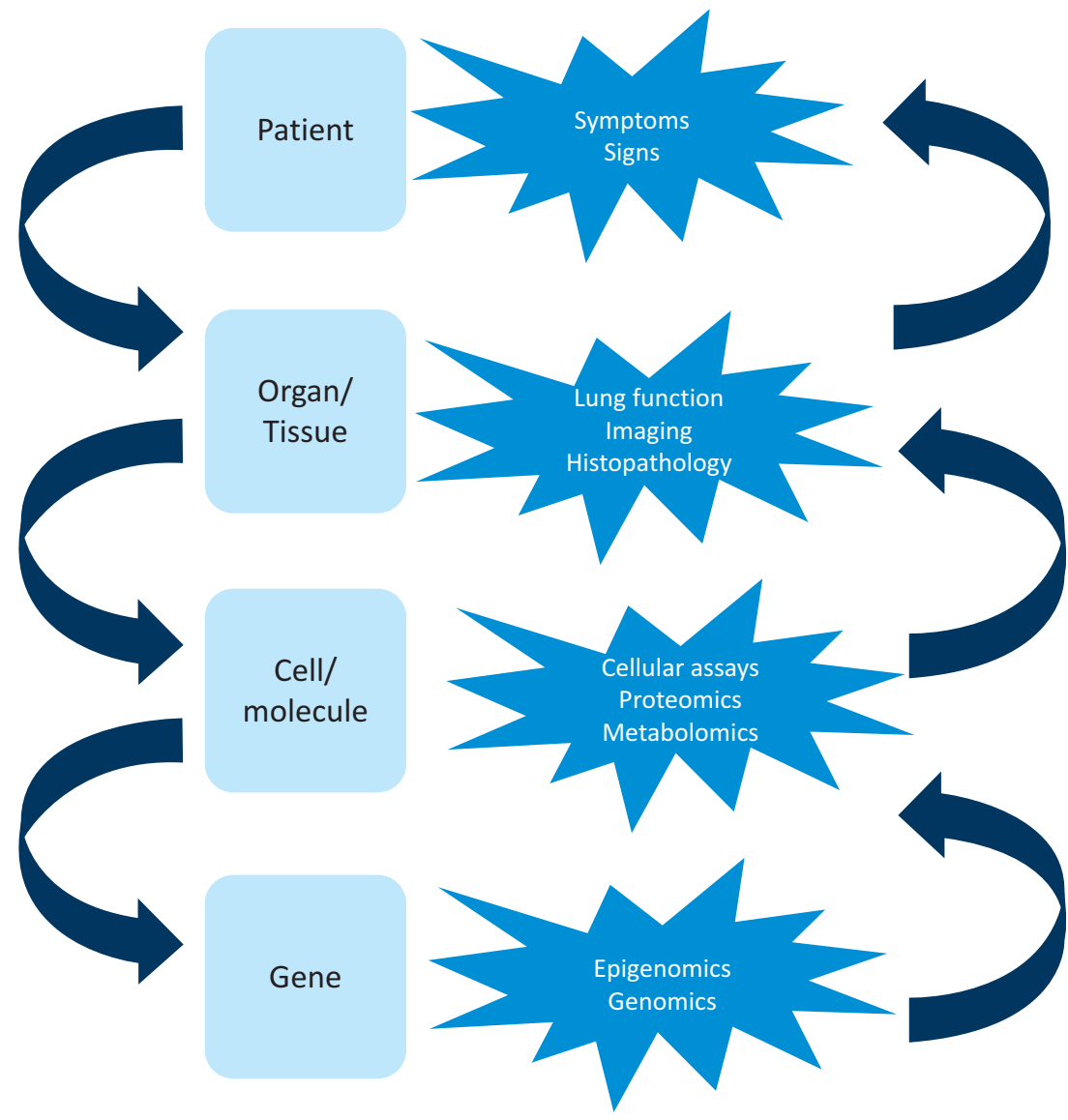

Fig 1. Phenotyping COPD. Chronic disease needs to be considered at multiple levels, as disease expression is dependent on the interactions with the environment across these levels. Likewise, the phenotype can be described by the integration of assessments made at the different levels.

between acquisition of a new pathogen versus persistent infection and the interplay with airway inflammation, lung function and symptoms are poorly understood. The strongest predictor of a COPD exacerbation is a previous history of an exacerbation, which has led to the concept of frequent versus infrequent exacerbators. ${ }^{3}$ This distinction is likely to reflect patients with different susceptibility to infection. To date, most attempts to phenotype COPD exacerbations have focussed on inflammatory biomarkers.

Hurst et al studied 36 plasma biomarkers in 90 patients with COPD, looking at paired baseline and exacerbation samples. ${ }^{4}$ C-reactive protein (CRP), a non-specific marker of infection, inflammation and injury, was the most selective but was not sufficient alone to confirm diagnosis of an exacerbation. However, when combined with one major symptom (increasing dyspnoea, sputum volume or purulence), $\mathrm{CRP} \geqslant 8 \mathrm{mg} / \mathrm{ml}$ performed better (95\% specificity and 57\% sensitivity for diagnosis of an exacerbation). None of the systemic biomarkers examined in this study were useful to predict severity of an exacerbation. Serum amyloid A (SAA) protein, like CRP, is increased at exacerbation onset compared with the stable state, but is more sensitive than CRP alone. When combined with SAA or CRP in this study, a major symptom did not improve prediction of severe episodes. These studies compared the stable state with exacerbations but did not fully classify the exacerbation event based on its potential aetiology.

Bafadhel et al recruited 145 patients into an observational study and assessed biomarkers from blood and serum in the stable state and during exacerbations for one year to define biomarkers to identify bacterial, viral or eosinophilic-associated exacerbations. ${ }^{5}$ In this study, $55 \%$ of exacerbations were associated with bacteria, $29 \%$ with viruses and $28 \%$ with sputum eosinophilia. The biomarkers that best identified these clinical phenotypes were sputum interleukin 1 beta (IL-1 $\beta$ ), serum C-X-C motif chemokine 10 (CXCL10) and percentage peripheral blood eosinophils, respectively. The observation that CXCL10 was the best biomarker for identifying viral exacerbations ( $80 \%$ sensitivity and $60 \%$ specificity) is consistent with previous findings that CXCL10 increases between baseline and COPD exacerbations due to human rhinovirus (HRV). Four biological exacerbation clusters were identified using unbiased cluster analysis; these validated the a priori aetiological groups: bacterial, viral, eosinophilic predominant and a fourth group described as 'pauci-inflammatory', as it was associated with limited changes in the inflammatory profile (Fig 2). These findings support the concept of different 'exacerbation phenotypes'. Interestingly, the eosinophilic predominant and bacterial-associated phenotypes were also observed in stable disease and predicted the future exacerbation phenotype. Patients experienced more bacterial exacerbations if their stable sputum samples contained bacteria (odds ratio 4.9 (95\% confidence interval (CI) 2.4 to 9.9)) and more eosinophilic exacerbations if eosinophilic inflammation was present in the stable state ( 2.7 (1.3 to 5.7$))$.

\section{Phenotype-specific management of stable COPD}

Within current practice, examples of phenotyping being used to target treatments to specific patient groups already exist. One example is the use of lung volume reduction surgery (LVRS) in the subgroup of patients with COPD who have radiological upper-lobe emphysema and a poor response to exercise therapy. This approach followed analysis of the National Emphysema Treatment Trial (NETT), which clearly demonstrated that this group benefited most from surgery. ${ }^{6}$ More recently, the antiinflammatory agent roflumilast (a phosphodiesterase-4 


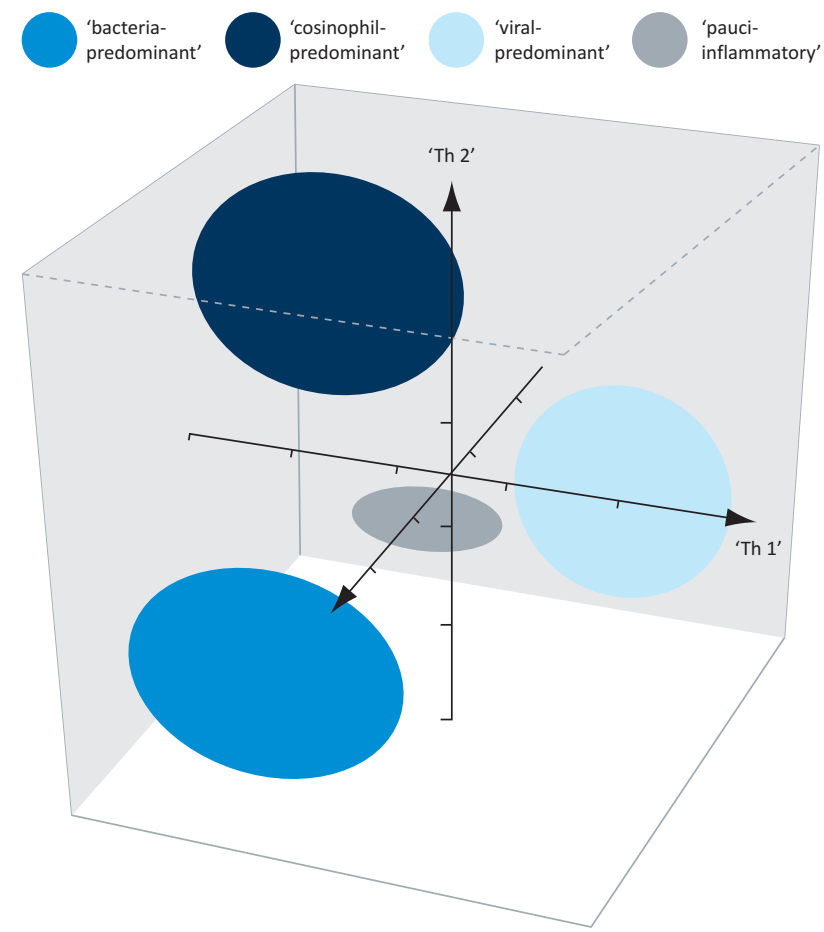

Fig 2. Proportional representation of biologic COPD clusters in threedimensional ellipsoids. Adapted from Hurst et al. 2006. ${ }^{4}$

inhibitor) has been investigated as a potential therapy for COPD. Initial results were disappointing, but post-hoc analyses of large clinical trials identified a subgroup that benefited more. Subsequent trials targeting roflumilast therapy to this COPD phenotype (with severe airways obstruction, history of exacerbations and chronic sputum production) have been encouraging, with a reduction in exacerbations as observed previously with inhaled corticosteroids. ${ }^{7}$

\section{Phenotype-specific management of COPD exacerbations}

Current evidence for the use of systemic corticosteroids and antibiotics in COPD exacerbations underscores the limited benefit of these therapies and their potential risks in an already vulnerable population. A Cochrane review collated data for 1,051 patients within 10 double-blind, randomised, controlled trials (RCTs) and compared systemic corticosteroids with placebo for the outcome of acute exacerbations of COPD. ${ }^{8}$ Systemic corticosteroids reduced the risk of treatment failure compared with placebo (OR 0.5 (95\% CI 0.36 to 0.69$)$ but no significant reduction in mortality was seen (OR 0.87 (95\% CI 0.45 to 1.66$)$ ). An early improvement in lung function was seen in the corticosteroid group $\left(\mathrm{FEV}_{1}\right.$ increased by $\left.140 \mathrm{ml}\right)$, although later outcomes less convincingly favoured corticosteroids; a reduction in hospital stay of 1.22 days was also seen in this group. Of concern, however, was the significant increase in adverse events observed in the corticosteroid arm (OR 2.33
( $95 \%$ CI 1.6 to 3.4)). The number needed to harm, or for one adverse event to occur, was six, with adverse events including hyperglycaemia, weight gain and insomnia.

Several reviews have examined the role of antibiotics in the treatment of acute exacerbations of COPD. Ram et al examined 917 patients in 11 RCTs of antibiotics versus placebo and found that antibiotics reduced mortality and treatment failure but were associated with an increased rate of diarrhoea. ${ }^{9}$ However, this study had several limitations, including differences in antibiotic choice and, importantly, a lack of control for other interventions that may have influenced outcomes (eg systemic corticosteroids and ventilatory support). Rothberg et al compared outcomes for hospitalised patients who received early antibiotic treatment for acute exacerbations of COPD with those in patients with late or no antibiotic treatment. ${ }^{10}$ In this retrospective analysis of almost 85,000 patients, those treated with antibiotics were less likely to be ventilated, had lower inpatient mortality and lower rates of readmission. Adverse effects of antibiotics were again highlighted and there was a notable increase in the rate of Clostridium difficile infection in the antibiotic-treated group. A review by Puhan et al, which included 13 placebo-controlled RCTs with 1,557 patients, noted significant heterogeneity across the trials due to exacerbation severity. In this review, antibiotics did not reduce treatment failure in outpatients with mild-to-moderate exacerbations; however, significant benefits for antibiotics were seen in patients with severe exacerbations in terms of reducing treatment failure (number needed to treat (NNT) 4) and mortality (NNT 14). A more recent placebo-controlled RCT was designed to try to eliminate some of the confounders influencing previously published systematic reviews and meta-analyses. In this study, 233 hospitalised patients with acute exacerbations of COPD were given systemic corticosteroids and randomised to doxycycline for one week or placebo. ${ }^{11}$ In terms of the primary study outcome at day 30, clinical success was similar in both groups; however, doxycycline was better than placebo at ensuring clinical success at day 10 and was superior in terms of clinical cure, microbiological outcome and improvement in symptoms at this earlier timepoint.

In order to maximise the benefit of corticosteroids and antibiotics in COPD exacerbations, it thus would be valuable to have biomarkers to identify phenotypes that respond to therapy and phenotypes for which there is the greatest risk of harm.

\section{Targeted steroid treatment for COPD}

In asthma and COPD, the presence of sputum eosinophilia is associated with a good response to corticosteroids. More importantly, targeted corticosteroid therapy aimed at normalising the sputum eosinophil count reduced exacerbations and hospital admissions in asthma and COPD. Siva et al randomised 82 patients with COPD to standard clinical care (in accordance with the British Thoracic Society's treatment guidelines) or targeted sputum management. The sputum management group 
had fewer severe exacerbations (mean reduction of $62 \%$ per patient per year), with no differences in the number of mild and moderate exacerbations or the average doses of oral or inhaled corticosteroid between the groups. ${ }^{12}$

This concept was recently extended to examine the effect of targeted/personalised treatment for eosinophilic airways inflammation at the time of an exacerbation event. Bafadhel et al performed a double-blind RCT using peripheral blood eosinophil count as a biomarker to guide oral corticosteroid treatment in outpatients with acute exacerbations of COPD. ${ }^{13}$ In this study, a safe reduction in inappropriate oral corticosteroid prescriptions was demonstrated. Intriguingly, patients without eosinophilia treated with systemic corticosteroids had more adverse events and a lower rate of recovery, which suggests that this biomarker identifies a group of patients who might benefit from and another group of patients harmed by corticosteroid therapy. This is a promising example of how treatments for acute exacerbations of COPD might be personalised and now needs replication and validation in larger patient groups, including those with severe acute exacerbations of COPD who are hospitalised for their exacerbations.

\section{Targeted antibiotic treatment for COPD}

It is desirable to be able to identify bacterial exacerbations reliably and to target antibiotics to this subgroup of patients. Procalcitonin (PCT) is a peptide produced in response to endotoxin and other mediators released in bacterial infections. It has been extensively evaluated as a 'bacterial infection' biomarker in many systemic infections, including lower respiratory tract infections and acute exacerbations of COPD. Current evidence suggests roles both in identifying bacterial acute exacerbations of COPD and in guiding the duration of antibiotic treatment. Schuetz et al performed a non-inferiority trial that recruited 1,359 patients with severe lower respiratory tract infections. Patients were randomised to antibiotics based on a PCT algorithm (predefined criteria for initiating/stopping antibiotics) or standard clinical treatment. Overall adverse events were similar in both groups but with shorter courses of antibiotics required in the PCT group (5.7 vs 8.7 days). This reduction in treatment duration was also seen in patients with acute exacerbations of COPD (PCT 2.5 days versus control 5.1 days). ${ }^{14}$ These results and those from other studies suggest that PCT has a role as a biomarker for acute bacterial exacerbations of COPD and that it can be used safely to reduce inappropriate antibiotics in acute exacerbations of COPD. We and others have demonstrated that CRP is a similarly sensitive and specific biomarker to identify patients who require antibiotics and may be a reliable, widelyavailable alternative to PCT.

\section{Conclusions}

In this review, we have discussed the limitations of the current symptomatic definition of acute exacerbations of COPD and the advances in biomarker studies to aid objective diagnosis of exacerbations. We have also discussed the importance of identifying clinically relevant exacerbation phenotypes, such as 'eosinophilic' and 'bacterial' exacerbations. It is hoped that further validation of biomarkers, including peripheral blood eosinophilia and CRP or PCT as biomarkers of eosinophilic and bacterial exacerbation phenotypes, respectively, will ultimately guide personalised treatment of exacerbations. Such personalised treatment should help to reduce inappropriate antibiotic and steroid use, along with their associated adverse effects.

We have also illustrated the heterogeneity encompassed within the current definition of COPD and the reasons why the respiratory community is seeking to phenotype patients. Attempts to phenotype COPD are at a relatively early stage, but international interest is ongoing, with data collection continuing in several large studies, such as the COPDGene study, the Evaluation of COPD Longitudinally to Identify Predictive Surrogate Endpoints (EClipSE) study, the SubPopulations and InteRmediate Outcome Measures In COPD Study (SPIROMICS) and the COPDMAP and AirPROM studies. By gaining a better understanding of COPD on many levels (organ, tissue, cell, molecule and genome), it will become possible to recognise the pathophysiology within a specific patient and ultimately to provide that patient with personalised treatment in the stable state and during exacerbations.

\section{References}

1 National Institute for Health and Clinical Excellence. Chronic obstructive pulmonary disease: management of chronic obstructive pulmonary disease in adults in primary and secondary care. Clinical guideline 101. London: NICE, 2010.

2 Brightling CE, Monteiro W, Ward R et al. Sputum eosinophilia and short-term response to prednisolone in chronic obstructive pulmonary disease: a randomised controlled trial. Lancet 2000;356:1480-5.

3 Hurst JR, Vestbo J Anzueto A et al. Susceptibility to exacerbation in chronic obstructive pulmonary disease. N Engl JMed 2010;363:112838.

4 Hurst JR, Donaldson GC, Perera WR et al. Use of plasma biomarkers at exacerbation of chronic obstructive pulmonary disease. Am J Respir Crit Care Med 2006;174:867-74.

5 Bafadhel M, McKenna S, Terry S et al. Acute exacerbations of chronic obstructive pulmonary disease: identification of biologic clusters and their biomarkers. Am J Respir Crit Care Med 2011;184:662-71.

6 Fishman A, Martinez F, Naunheim K et al. A randomized trial comparing lung-volume-reduction surgery with medical therapy for severe emphysema. N Engl J Med 2003;348:2059-73.

7 Calverley PM, Rabe KF, Goehring UM et al. Roflumilast in symptomatic chronic obstructive pulmonary disease: two randomised clinical trials. Lancet 2009;374:685-94.

8 Walters JA, Gibson PG, Wood-Baker R et al. Systemic corticosteroids for acute exacerbations of chronic obstructive pulmonary disease. Cochrane Database Syst Rev 2009;(1):CD001288.

9 Ram FS, Rodriguez-Roisin R, Granados-Navarrete A et al. Antibiotics for exacerbations of chronic obstructive pulmonary disease. Cochrane Database Syst Rev 2006;(2):CD004403.

10 Rothberg MB, Pekow PS, Lahti M et al. Antibiotic therapy and treatment failure in patients hospitalized for acute exacerbations of chronic obstructive pulmonary disease. JAMA 2010;303:2035-42. 
11 Daniels JM, Snijders D, de Graaff CS et al. Antibiotics in addition to systemic corticosteroids for acute exacerbations of chronic obstructive pulmonary disease. Am J Respir Crit Care Med 2010;181:150-7.

12 Siva R, Green RH, Brightling CE et al. Eosinophilic airway inflammation and exacerbations of COPD: a randomised controlled trial. Eur Respir J 2007;29:906-13.

13 Bafadhel M, McKenna S, Terry S et al. Blood eosinophils to direct corticosteroid treatment of exacerbations of COPD: a randomized placebo controlled trial. Am J Respir Crit Care Med 2012; 23 Mar: [epub ahead of print].
14 Schuetz P, Christ-Crain M, Thomann R et al. Effect of procalcitoninbased guidelines vs standard guidelines on antibiotic use in lower respiratory tract infections: the ProHOSP randomized controlled trial. JAMA 2009;302:1059-66.

Address for correspondence: Prof CE Brightling, Institute for Lung Health, Clinical Sciences Wing, University Hospitals of Leicester NHS Trust, Glenfield Hospital, Groby Road, Leicester LE3 9QP.

Email: ceb17@le.ac.uk 